\title{
Modeling and Automating of RCS's Lab Tracker
}

\author{
Mohamed Galal and Jian-Yu Wang
}

\begin{abstract}
Radar cross section measurement must be precise and accurate especially for tracking small radar cross section targets (e.g. tracks on Fighters); other targets of interest may be aircraft, tanks, or ships. As a consequence, the need of having more accurate evaluation for the Radar Cross Section (RCS) of the targets is required. This paper describes and demonstrates the modeling/automation of the RCS's antenna holder movements. In order to suppress the unwanted manmade error, fuzzy logic controller was used, to have better target tracking while measurement process. Taking into account the fuzzy nature of human decision making processes and real-time properties, this paper has established different fuzzy controllers then tested and compared with a crisp decision system. The first one has been developed using an expert knowledge, the second one was learned from the recorded videos.
\end{abstract}

Index Terms-Fuzzy logic control, multi-input/ multi output, nonlinear system, RCS lab measurements enhancement, and video guidance.

\section{INTRODUCTION}

In the existing outdoor RCS lab, science is specifically interested in measuring the radar-cross-section RCS of an (aircraft fighter/civilian, Tanks, Ships, Chaffs, and Rams). Correctly identifying and evaluateing the target's RCS, more accurate information can be provided for the command and guidance systems to precisely attack the most threatening targets, or provide more help for RCS reduction scientists [1]-[8].

The main idea of obtaining the RCS of any target is measuring the target from each aspect angle compared to a reference target at the same field. The need to measure at each aspect angle leads to put both the transmitting, receiving antennas and the digital camera on a turn table. Vision guidance is accomplished with a CCD camera with a zoom lens. The data is collected through a commercial tracking device, communicating to the computer. In the existing lab, Vision tracking was accomplished by using a joystick to track the target maneuvers as shown in figure (1). To track while measurement process, in order to suppress the unwanted man made error, an online nonlinear controller for nonlinear tracking should be used [9], [10].

The control technique used for the turntable (antenna holder) used on RCS lab system must provide fast and precise performance. Poor tracking performance during measurement will result in large miss evaluation which may cause misleading results. The equations describing the RCS's lab turntable system under consideration are highly nonlinear.

Manuscript received April 14, 2016; revised June 14, 2016.

The authors are with the School of Automation, Nanjing University of Science and Technology, Nanjing, China (e-mail: Mohamed.Galal@ njust.edu.cn, jianyu_wang2000@163.com).
Accurate nonlinear control is required in order to precisely keep the LOS between the RCS lab's antenna and the target to be measured [9]. Generally, an accurate nonlinear model of a system like that RCS's antenna holder system is difficult to find, and parameter values obtained from system identification may be only approximated values.

One of the intelligent techniques, is fuzzy logic and had been developed by Zadeh [10], [11] is applied for controller design in many applications [10], [11]. A fuzzy logic controller (FLC) was proved analytically to be equivalent to a nonlinear PI controller when a nonlinear defuzzification method is used [12]. Also, the results from the comparison of conventional and fuzzy logic control techniques in the form of FLC [14], [15] and fuzzy compensator [16], [17] showed that fuzzy logic can reduce the effects of nonlinearity and improve the performance of the system.

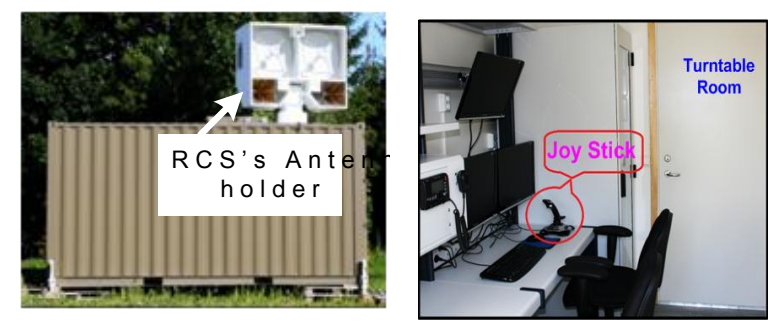

Fig. 1. Outdoor RCS lab.

This paper uses Dinavit-Hartinberg, and Lagrange equations to arrange the axes and drive the equations of motion in both the azimuth and elevation plans, then uses fuzzy logic control to automate the tracking process of the RCS's Lab antenna holder. The advantages of the fuzzy logic system are that multiple types of input such as from vision and sonar sensors as well as stored map information can be used in guidance process.

Heuristic knowledge is applied to define fuzzy membership functions and rules. The membership functions and rules are then modified. The rest of this article is organized as follows. Outdoor RCS Lab theory of operation, Dynamic model of the turntable system is derived in Section two. A FLC control algorithm is proposed in Section three. In section four, The simulation model and results are illustrated. Then conclusion will be given in Sections five.

\section{RCS LAB SYSTEM}

\section{A. Lab Description}

The main idea of obtaining the RCS of any target is measuring the target from each aspect angle compared to a reference target at the same circumstances. The need to measure at each aspect angle leads to put both the transmitting, receiving antennas and the digital camera on a turn table as 
shown in Fig. 2 and Fig. 3. Vision guidance is accomplished with a CCD camera with a zoom lens. The data is collected through a commercial tracking device, communicating to the computer. In the existing lab, Vision tracking was accomplished by using a joystick to track the target maneuvers.

Software on the radar control PC is used to setup the measurements requirements such as (Frequency, Transmitter/Receiver Polarization, Pulse Repetition Frequency, $\min / \max$ range of measurements, Calibration value, ..etc). These settings are sent through the LAN switch to the Signal generator then to both the Transmitter/Receiver. Analog to digital converter (ADC) PC samples, stores, and processes both transmitted/received signals. Video Controller PC interfaces with CCD and the Joystick then controlling the antenna position by the turntable controller through the LAN switch. Figure (2) shows both processing/controlling, and turntable room. This figure shows the three computers, LAN switch, Signal generator, and turntable room. Moving the antenna to track the moving target by the joystick is difficult and is the reason of insufficient measurements. This leads to the need of automatically move the turntable to track the moving target.

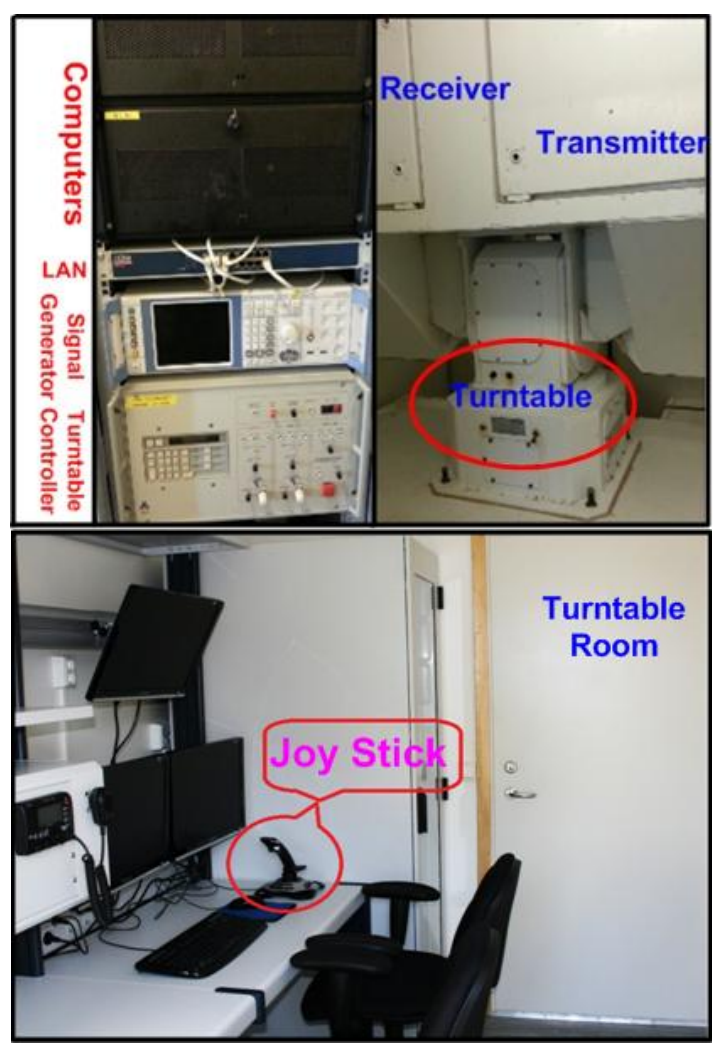

Fig. 2. Processing, controlling, and turntable rooms.

So the importance of having automatic movements of the turntable to track the target, which prohibit misleading RCS measurements and evaluation, comes to light.

The turn table shown in figure (4), consists of two revolute joints (the first revolute joint is for Elevation movement and the second is for Azimuth) with two motors, the antenna-holder of length " $\mathrm{L}$ " is attached to the motor number "2".

\section{B. System Model}

The dynamical analysis of the RCS's Lab antenna holder investigates a relation between the joint torques/forces applied by the actuators, and the position, velocity and acceleration of the RCS turntable with respect to time. Turntable manipulators have complex non-linear dynamics that might make applying accurate and robust control difficult. The turntable system illustrated in Figure (4) consists of two revolute joints derived with two motors. According to Dinavit_Hartinberg convention the coordinate frames are considered and rearranged.
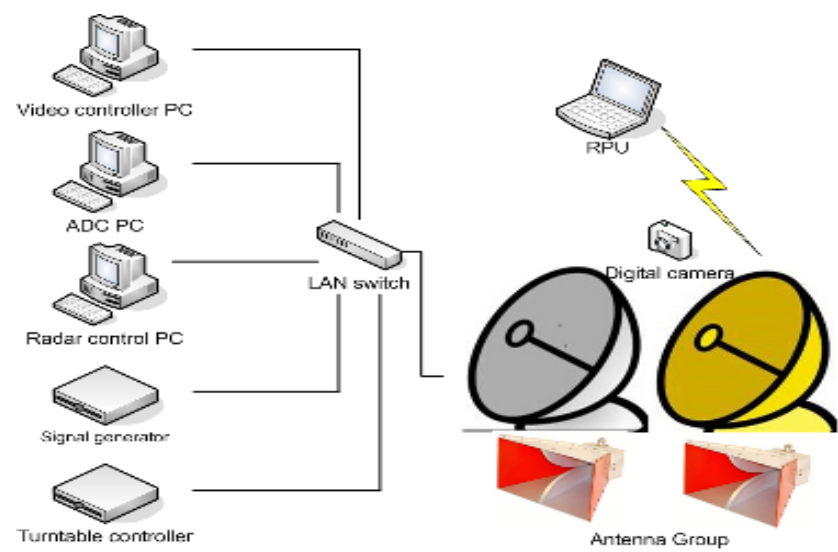

Fig. 3. RCS lab basic blocks.

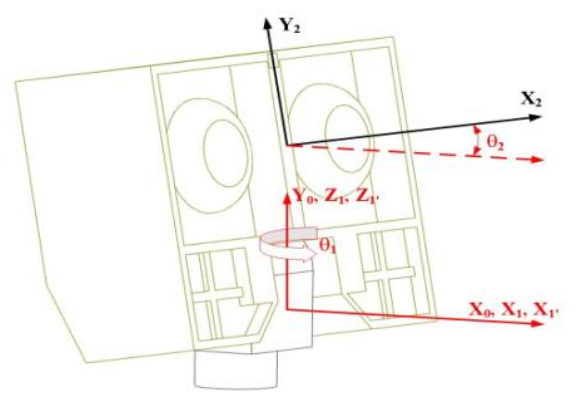

Fig. 4. Coordinate frames according to Dinavit-Hartinberg convention

According to Dinavit-Hartinberg convention the coordinate frames are considered and rearranged as shown in figure (4). The joint parameters and the transformation matrices can be defined as shown in Table I.

\begin{tabular}{c|c|c|c|c}
\multicolumn{5}{c}{ TABLE I: SYSTEM'S JOINT PARAMETERS } \\
\hline \hline Joint & $\theta$ & $d$ & $a$ & $\alpha$ \\
\hline 1 & $\theta_{1}$ & 0 & 0 & 90 \\
\hline$I^{\prime}$ & 0 & 0 & $a$ & 0 \\
\hline 2 & $\theta_{2}$ & 0 & $L$ & 0 \\
\hline \hline
\end{tabular}

where the joint parameters $\theta, \mathrm{d}$, a and $\alpha$ are defined with DH convention using the transformation matrix defined in the next equation,

$\theta_{1} \quad$ angular position of motor " 1 ".

$\theta_{2}$ angular position of motor " 2 ".

a distance between the two motors in the $X_{1}$ direction.

$\mathrm{L}$ antenna-holder length.

Then the transformation from frame to another can be defined 
as,

$$
\begin{gathered}
\underline{T}_{01}=\left[\begin{array}{cccc}
c_{1} & 0 & s_{1} & 0 \\
s_{1} & 0 & -c_{1} & 0 \\
0 & 1 & 0 & 0 \\
0 & 0 & 0 & 1
\end{array}\right], \quad \underline{T}_{11}=\left[\begin{array}{cccc}
1 & 0 & 0 & a \\
0 & 1 & 0 & 0 \\
0 & 0 & 1 & 0 \\
0 & 0 & 0 & 1
\end{array}\right], \\
\underline{T}_{12}=\left[\begin{array}{cccc}
c_{2} & -s_{2} & 0 & L c_{2} \\
s_{2} & c_{2} & 0 & L s_{2} \\
0 & 0 & 1 & 0 \\
0 & 0 & 0 & 1
\end{array}\right]
\end{gathered}
$$

where

$\underline{T}_{i j}$ Transformation matrix from frame " $\mathrm{j}$ " to frame "ij".

$c_{1}, s_{1} \quad \cos \theta_{1}$ and $\sin \theta_{1}$ respectively.

$c_{2}, s_{2} \quad \cos \theta_{2}$ and $\sin \theta_{2}$ respectively.

The transformation from $x_{1}^{\prime} y_{1}^{\prime} z_{1}^{\prime}$ frame to $x_{0} y_{0} z_{0}$ frame can be defined by chain rule as,

$$
T_{01}=\underline{T}_{01} \underline{T}_{11}=\left\lfloor\begin{array}{cccc}
c_{1} & 0 & s_{1} & a c_{1} \\
s_{1} & 0 & -c_{1} & a s_{1} \\
0 & 1 & 0 & 0 \\
0 & 0 & 0 & 1
\end{array}\right\rfloor
$$

Similarly, the transformation matrix from $x_{2} y_{2} z_{2}$ frame to $x_{0} y_{0} z_{0}$ frame can be represented as follows,

$$
\begin{aligned}
\underline{T}_{02}=\underline{T}_{01} \underline{T}_{11} \underline{T}_{12}=\underline{T}_{01} \underline{T}_{12} \\
\left.\underline{\mathrm{T}}_{02}=\left\lfloor\begin{array}{cccc}
\mathrm{c}_{1} & 0 & \mathrm{~s}_{1} & \mathrm{ac}_{1} \\
\mathrm{~s}_{1} & 0 & -\mathrm{c}_{1} & \mathrm{as}_{1} \\
0 & 1 & 0 & 0 \\
0 & 0 & 0 & 1
\end{array}\right\rfloor \mid \begin{array}{cccc}
\mathrm{c}_{2} & -\mathrm{s}_{2} & 0 & \mathrm{Lc}_{2} \\
\mathrm{~s}_{2} & \mathrm{c}_{2} & 0 & \mathrm{Ls}_{2} \\
0 & 0 & 1 & 0 \\
0 & 0 & 0 & 1
\end{array}\right\rfloor
\end{aligned}
$$

The absolute position of a small segment " $d x$ " of the antenna-holder as shown in figure (5) in terms of the joint parameters in table " 1 " can be expressed as,

$$
\underline{r}_{2}=\underline{T}_{02} r_{2 / 2}
$$

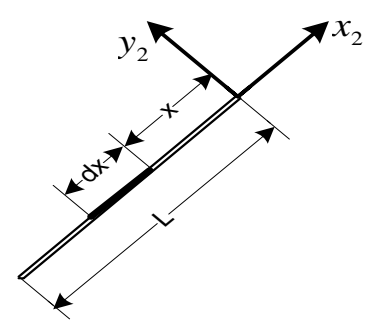

Fig. 5. Absolute position of a small segment " $d x "$.

where

$\underline{r}_{2 / 2} \quad$ local position of the small element " $d x$ " with respect to $x_{2} y_{2} z_{2}$ frame (frame $2^{\prime}$ ).

$$
\underline{R}_{2 / 2}=\left[\begin{array}{llll}
x & 0 & 0 & 1
\end{array}\right]^{T}
$$

$\underline{T}_{02} 4 * 4$ transformation matrix from frame " 2 " to frame "0" $x_{0} y_{0} z_{0}$

$$
\begin{aligned}
\underline{r}_{2} & \left.=\left\lfloor\begin{array}{cccc}
c_{1} c_{2} & -c_{1} s_{2} & s_{1} & L c_{1} c_{2}+a c_{1} \\
s_{1} c_{2} & -s_{1} s_{2} & -c_{1} & L s_{1} c_{2}+a s_{1} \\
s_{2} & c_{2} & 0 & L s_{2} \\
0 & 0 & 0 & 1
\end{array}\right\rfloor \mid \begin{array}{l}
x \\
0 \\
0 \\
1
\end{array}\right] \\
& =\left[\begin{array}{llll}
r_{x} & r_{y} & r_{z} & 1
\end{array}\right]^{T}
\end{aligned}
$$

where

$r_{x}, r_{y}, r_{z}$ Components of the absolute position of segment " $d x "$ at distance " $x "$ in the negative $x_{2}$ direction.

The first derivative of equation (2) represents the absolute velocity which is defined as,

$$
\dot{\underline{r}}_{2}=\left[\begin{array}{c}
-(x+L) c_{1} s_{2} \dot{\theta}_{2}-(x+L) s_{1} c_{2} \dot{\theta}_{1}-a s_{1} \dot{\theta}_{1} \\
-(x+L) s_{1} s_{2} \dot{\theta}_{2}-(x+L) c_{1} c_{2} \dot{\theta}_{1}-a c_{1} \dot{\theta}_{1} \\
(x+L) c_{2} \dot{\theta}_{2} \\
0
\end{array}\right]
$$

\section{Dynamic Modeling}

A mathematical model of the Azimuth-Elevation movement manipulator system including a dynamic model of a rigid has been studied. The dynamic modeling has been introduced using Lagrange's equation of motion and the direct Kinematics of the Azimuth-Elevation.

Lagrangian dynamic modeling is used to derive the equation of motion due to its Straight forward and its systematic nature. Also, Lagrange's equation is especially suited for modeling of complex systems.

The generalized coordinates have been chosen to describe the Elevation movement motion and to apply Lagrangian dynamic properly. The generalized coordinate vector is defined by $2 * 1$ vector $\left[\begin{array}{ll}\theta_{1} & \theta_{2}\end{array}\right]^{T}$.

Hence, Lagrange's equations for the system are defined in general form as,

$$
\frac{d}{d t}\left(\frac{\partial T}{\partial \dot{q}_{i}}\right)-\frac{\partial T}{\partial q_{i}}+\frac{\partial V}{\partial q_{i}}+\frac{\partial D}{\partial \dot{q}_{i}}=Q_{i}
$$

where

$T$ Total kinetic energy of the system.

$V$ Total potential energy of the system.

$D$ Dissipation function due to damping.

$Q_{i}$ Generalized force corresponding to i-coordinate.

Then, the total kinetic energy, potential energy, dissipation function (if we have damping) and the virtual work of the system must be obtained to apply Lagrangian dynamic modeling.

\section{Kinetic Energy}

The total kinetic energy of the system consists of four main terms, the kinetic energy for the elevation movement $T_{E}$, the kinetic energy of the Azimuth $T_{A}$, the kinetic energy of the two rotors of the motors $T_{R_{1}}$ and $T_{R_{2}}$, and the kinetic energy of the payload at the end effector of the 
antenna-holder.

Kinetic Energy of the Elevation movement $T_{E}$

The kinetic energy of the Elevation movement is due to its revolution around its center:

where

$m_{1}$ mass of the Elevation movement.

a radius of motion.

$\dot{\theta}_{1} \quad$ angular speed of Elevation movement.

Kinetic Energy of the elevation motion $T_{E}$

The kinetic energy of the antenna-holder is due to the distributed of its mass along the length " $\mathrm{L}$ " is defined by:

$$
T_{E}=\frac{1}{2} \int_{A r m} \dot{r}_{2}^{T} \dot{r}_{2} d m
$$

where

$$
d m=\rho A d x
$$

A Cross-section area of the antenna holder.

$\rho$ material density of the Gun Turret-Barrel system.

From equation (3) the absolute velocity can be used as,

$$
\dot{r}_{2}^{T} \dot{r}_{2}=(x+L)^{2} \dot{\theta}_{2}^{2}+\left[(x+L) c_{2}+a\right]^{2} \dot{\theta}_{1}^{2}
$$

Hence,

$$
\begin{array}{r}
T_{A}=\frac{1}{2} \rho A \int_{-L}^{0} \dot{r}_{2}^{T} \dot{r}_{2} d x \\
T_{A}=\frac{1}{2}(\rho A L) \frac{L^{2}}{3} \dot{\theta}_{2}^{2}+\frac{1}{2} \rho A L\left(\frac{L^{2}}{3} c_{2}^{2}+a L c_{2}+a^{2}\right) \dot{\theta}_{1}^{2}
\end{array}
$$

\section{Kinetic Energy of the two Motors}

The kinetic energy of the motor is due to the rotation of its rotor of moment of inertia $I_{R}$ by an angular velocity $\omega$ can be expressed by:

$$
T_{R_{1}}=\frac{1}{2} I_{R_{1}} \underline{\omega}_{R_{1}}^{T} \underline{\omega}_{R_{1}}
$$

where $\underline{\omega}_{R_{1}}$ is angular velocity vector of motor " 1 ".

The angular coordinate vector of motor " 1 " with respect to frame " 1 " can be defined by:

$$
\underline{\theta}_{1 / 1}=\left[\begin{array}{llll}
0 & \theta_{1} & 0 & 1
\end{array}\right]^{T}
$$

By tacking the $1^{\text {st }}$ derivative to obtain the angular velocity vector of motor " 1 " with respect to frame " 1 "gives:

$$
\underline{\omega}_{1 / 1}=\left[\begin{array}{llll}
0 & \dot{\theta}_{1} & 0 & 1
\end{array}\right]^{T}
$$

Hence, the absolute angular velocity can be defined using the transformation matrix $\underline{T}_{01}$ which is defined earlier.

$$
\underline{\omega}_{1}=\underline{T}_{01} \underline{\omega}_{1 / 1}=\left\lfloor\begin{array}{cccc}
c_{1} & 0 & s_{1} & 0 \\
s_{1} & 0 & -c_{1} & 0 \\
0 & 1 & 0 & 0 \\
0 & 0 & 0 & 1
\end{array}\right\rfloor\left\lfloor\begin{array}{c}
0 \\
\dot{\theta}_{1} \\
0 \\
0
\end{array}\right\rfloor=\left[\begin{array}{llll}
0 & 0 & \dot{\theta}_{1} & 0
\end{array}\right]^{T}
$$

Then, the kinetic energy of the motor " 1 " is expressed as,

$$
\therefore T_{R_{1}}=\frac{1}{2} I_{R_{1}} \dot{\theta}_{1}^{2}
$$

where

$T_{R_{1}}$ Kinetic energy of rotor " 1 "

$I_{R_{1}}$ Mass moment of inertia of rotor " 1 "

$\underline{\omega}_{R_{1}}=\underline{\omega}_{1}$ angular velocity vector of the first motor.

$$
T_{R_{2}}=\frac{1}{2} I_{R_{2}} \underline{w}_{R_{2}}^{T} \underline{w}_{R_{2}}+\frac{1}{2} m_{R_{2}} \underline{\dot{r}}_{R_{2}} \underline{\dot{r}}_{R_{2}}
$$

The angular coordinate vector of motor " 2 " with respect to frame " 1 " can be represented by:

$$
\underline{\theta}_{2 / 1}=\left[\begin{array}{llll}
0 & 0 & \theta_{2} & 1
\end{array}\right]^{T}
$$

The first derivative of this angular coordinate vector of motor " 2 " with respect to frame " 1 "

$$
\underline{\omega}_{2 / 1}=\left[\begin{array}{llll}
0 & 0 & \dot{\theta}_{2} & 1
\end{array}\right]^{T}
$$

Using the transformation matrix $T_{01}$ to transform the angular velocity from frame "1" to frame " 0 " as follows $\underline{\omega}_{2}=\underline{T}_{01} \underline{\omega}_{2 / 1}=\left[\begin{array}{llll}s_{1} \dot{\theta}_{2} & -c_{1} \dot{\theta}_{2} & 0 & 0\end{array}\right]^{T}$

The angular velocity of rotor " 2 " is represented by the summation of angular velocities of the two motors in frame "0"

$$
\underline{\omega}_{R_{2}}=\underline{\omega}_{1}+\underline{\omega}_{2}
$$

Hence,

$$
\underline{\dot{r}}_{R_{2}}^{T} \dot{\dot{r}}_{R_{2}}=a^{2} \dot{\theta}_{1}^{2}
$$

From equation (10), (11) in equation (9)

$$
T_{R_{2}}=\frac{1}{2} I_{R_{2}} \dot{\theta}_{2}^{2}+\frac{1}{2} m_{R_{2}} \dot{\theta}_{1}^{2}
$$

\section{Kinetic Energy of the Payload}

The position of the end effectors of the system (the tip point of the antenna-holder) can represent the position of the payload and can be defined with respect to $x_{2} y_{2} z_{2}$ frame (frame 2) as follows,

$$
\begin{aligned}
& \underline{r}_{p / 2}=\left[\begin{array}{llll}
0 & 0 & 0 & 1
\end{array}\right]^{T} \\
& \underline{r}_{p}=T_{02} \underline{r}_{p / 2}=\left[\begin{array}{llll}
L c_{1} c_{2}+a c_{1} & L s_{1} c_{2}+a s_{1} & L s_{2} & 1
\end{array}\right]^{T}
\end{aligned}
$$

The absolute velocity of the payload $\underline{\dot{r}}_{p}$,

$$
T_{p}=\frac{1}{2} m_{p} \dot{\underline{r}}_{p}^{T} \underline{\dot{r}}_{p}=\frac{1}{2} m_{p}\left(L c_{2}+a^{2}\right) \dot{\theta}_{1}^{2}+\frac{1}{2} m_{p} L^{2} \dot{\theta}_{2}^{2}
$$

where $m_{p}$ is the payload's mass.

From equations $(7,8,12$, and 14) the total kinetic energy can be summarized as, 


$$
T=T_{T}+T_{R_{1}}+T_{R_{2}}+T_{p}
$$

\section{Potential Energy}

The potential energy due to gravity of the antenna-holder, the payload and motor " 2 " represent the total potential energy of the system as the strain energy of the antenna-holder is neglected due to it is considered as a rigid body.

Gravitational potential energy of the antenna-holder

$$
V_{A}=\int_{A r m} \underline{r}_{2}^{T} g d m
$$

where

$V_{A}$ Gravitational potential energy of the antenna-holder.

$\underline{g}$ Gravitational acceleration vector.

$$
\underline{g}=\left[\begin{array}{llll}
0 & 0 & -9.81 & 0
\end{array}\right]^{T}
$$

$\underline{r}_{2} \quad$ Absolute position vector of a small mass

$d m=\rho A d x$, which was defined earlier in equation (2).

$$
V_{A}=-9.81 \rho A \frac{L^{2}}{2} s_{2}
$$

Gravitational potential energy of the payload

$$
V_{p}=m_{p} \underline{r}_{p}^{T} g=-9.81 m_{p} L s_{2}
$$

From equations (2.16) and (2.17), the total potential energy is defined as,

$$
V=V_{A}+V_{p}=-9.81 \rho A \frac{L^{2}}{2} s_{2}-9.81 m_{p} L s_{2}
$$

\section{Virtual Work}

For a system without any external applied force at the end effector, the generalized force can be obtained from the virtual work of the system,

$$
\delta W=\delta \underline{q} \underline{Q}
$$

The virtual work of the considered system is given by: $\delta W=M_{1} \delta \theta_{1}+M_{2} \delta \theta_{2}$

Then,

$$
\underline{Q}=\left\lfloor\begin{array}{l}
M_{1} \\
M_{2}
\end{array}\right\rfloor
$$

where

$\underline{Q}$ the generalized force.

$M_{1}$ applied torque at motor " 1 ".

$M_{2}$ applied torque at motor " 2 ".

Lagrange's Equation of Motion

Lagrange's equation of motion is applied to give two equations of motion, one to describe the turntable motion by angular coordinate $\theta_{1}$ and the second one to describe the motion of the antenna-holder by angular coordinate $\theta_{2}$.

Turntable's Equation of Motion

Lagrange's equation is applied for generalized coordinate.
Then, the equation of motion which describe the turntable motion is obtained by applying Lagrange's equation in the following form,

$$
\frac{d}{d t}\left(\frac{\partial T}{\partial \dot{\theta}_{1}}\right)-\frac{\partial T}{\partial \theta_{1}}+\frac{\partial V}{\partial \theta_{1}}=Q_{1}
$$

$$
\begin{aligned}
& \mid\left(\begin{array}{l}
\left.\frac{\rho A L^{3}}{3}+m_{p} L^{2}\right) c_{2}^{2}+\left(\rho A L^{2} a+2 m_{p} L a\right) c_{2} \\
+\left(\rho A L a^{2}+I_{R_{1}}+m_{R_{2}} a^{2}+m_{p} a^{2}+\frac{1}{2} m_{1} R_{1}^{2}\right)
\end{array}\right) \ddot{\theta}_{1} \\
& -\left[2\left(\frac{\rho A L^{3}}{3}+m_{p} L^{2}\right) c_{2} s_{2}+\left(\rho A L^{2} a+2 m_{p} L a\right) s_{2}\right] \dot{\theta}_{2} \dot{\theta}_{1}=M_{1}
\end{aligned}
$$

Antenna-holder's Equation of Motion

For the second generalized $q_{2}=\theta_{2}$ Lagrange's equation of motion can be applied as,

$$
\frac{d}{d t}\left(\frac{\partial T}{\partial \dot{\theta}_{2}}\right)-\frac{\partial T}{\partial \theta_{2}}+\frac{\partial V}{\partial \theta_{2}}=Q_{2}
$$

Hence,

$$
\begin{aligned}
& \left(\frac{\rho A L^{3}}{3}+I_{R_{2}}+m_{p} L^{2}\right) \ddot{\theta}_{2} \\
& +\left(\frac{\rho A L^{3}}{3} c_{2} s_{2}+m_{p} L^{2} c_{2} s_{2}+\frac{\rho A L^{2} a}{2} s_{2}+m_{p} \text { Las }_{2}\right) \dot{\theta}_{1}^{2} \\
& -9.81\left(\frac{\rho A L^{2}}{2}+m_{p} L\right) c_{2}=M_{2}
\end{aligned}
$$

The equations (20) and (21) describe the equations of motion of the system which consists of an antenna-holder based on a turntable.

\section{FuZZY LOGIC CONTROLlER DESIGN}

While conventional controllers depend on the accuracy of the system model and parameters, FLCs use a different approach to control the system. Fuzzy logic controller (FLC) is one of the most important applications of fuzzy logic theory. This controller can be used to control nonlinear, uncertain, and noisy systems. Instead of using system model, the operation of a FLC is based on heuristic knowledge and linguistic descriptions to the performed task. The effects from inaccurate parameters and models are reduced because a FLC does not require a system model. However, building a FLC from the ground-up may not provide good result than a conventional controller if there is not enough knowledge of the system. Therefore, in this paper, the FLC is initially constructed as a-prior knowledge in the process. The performance of the FLC is then improved by adjusting the rules and membership functions. These design procedure are described as follows. 


\section{A. Procedure 1: Defining Inputs, Outputs, and Universe of} Discourse

To apply heuristic knowledge in the FLC, inputs, outputs, and universe of discourse are defined first. The inputs are the error (e) between the reference position $\left(\theta_{r}\right)$ and the actual position $\left(\theta_{a}\right)$, and the change in error $(\dot{e})$. The output is the change in armature voltage (CU). Figure (5) describes inputs, output, and FLC block diagram.

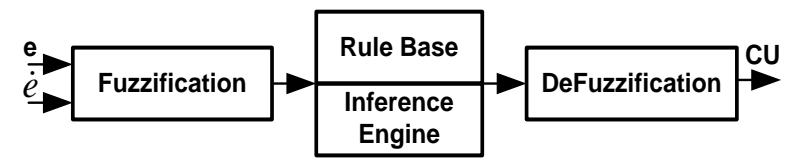

Fig. 5. Block diagram of the FLC.

\section{B. Procedure 2: Fuzzy Membership Functions And rules}

To perform fuzzy computation, inputs and output must be converted from numerical or crisp value into linguistic forms. The terms such as "Small" and "Big" are used to quantize inputs and output values to linguistic terms that used to represent input and output values are defined by seven fuzzy variables tabulated at Table II. Fuzzy membership functions are used as tools to convert crisp values to linguistic terms forms.

TABLE II: FUZZY LINGUISTIC TERMS

\begin{tabular}{l|l}
\hline \hline Term & Definition \\
\hline NB & Negative big \\
\hline NM & Negative medium \\
\hline NS & Negative small \\
\hline ZE & zero \\
\hline PS & Positive small \\
\hline PM & Positive medium \\
\hline PB & Positive big \\
\hline
\end{tabular}

TABLE III: INITIAL RULES

\begin{tabular}{c|c|c|c|c|c|c|c}
\hline \hline & NB & NM & NS & ZE & PS & PM & PB \\
\hline NB & NB & NB & NB & NB & NM & NS & ZE \\
\hline NM & NB & NB & NB & NM & NS & ZE & PS \\
\hline NS & NB & NB & NM & NS & ZE & PS & PM \\
\hline ZE & NB & NM & NS & ZE & PS & PM & PB \\
\hline PS & NM & NS & ZE & PS & PM & PB & PB \\
\hline PM & NS & ZE & PS & PM & PB & PB & PB \\
\hline PB & ZE & PS & PM & PB & PB & PB & PB \\
\hline \hline
\end{tabular}

TABLE IV: MODIFIED RULES

\begin{tabular}{c|c|c|c|c|c|c|c}
\hline \hline & NB & NM & NS & ZE & PS & PM & PB \\
\hline NB & NB & NB & NB & NB & ZE & PM & PM \\
\hline NM & NB & NB & NB & NM & PS & PM & PB \\
\hline NS & NB & NB & NM & NS & PM & PM & PB \\
\hline ZE & NB & NM & NS & ZE & PB & PB & PB \\
\hline PS & NM & NS & ZE & PS & PM & PB & PB \\
\hline PM & NS & ZE & PS & PM & PB & PB & PB \\
\hline PB & ZE & PS & PM & PB & PB & PB & PB \\
\hline \hline
\end{tabular}

A fuzzy membership function can contain several fuzzy sets depending on how many linguistic terms. In this paper, seven fuzzy sets are obtained by applying the seven linguistic terms. The number for indicating how much a crisp value can be a member in each fuzzy set is called a degree of membership. One crisp value can be converted to be partly in many fuzzy sets, but the membership degree in each fuzzy set may be different. In order to define fuzzy membership function, designers can choose many different shapes based on their preference or experience. The popular shapes are triangular and trapezoidal because these shapes are easy to represent designer's ideas and require low computation time. For performing fine-tuning to improve the efficient of the controller, the adjacent of each fuzzy set value should overlap by about $25 \%$ [19]. The membership functions are illustrated in Fig. 4. In equation (1), we see that the equations of motion are in a nonlinear form. We ought to use FLC to overcome this nonlinearity of the equation of motion of the system to be controlled. Instead of using mathematical formulas, a FLC uses fuzzy rules to make a decision and generate the control effort [20]. The rules are in the form of IF-THEN statements. For example, IF the error (e) is equal to Positive Big (PB) and the change in error $(\dot{e})$ is equal to Positive.

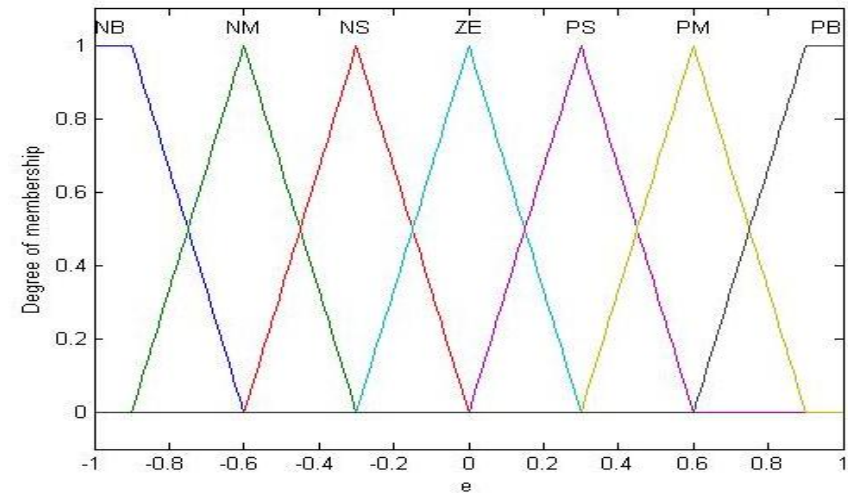

Fig. 6. Membership functions.

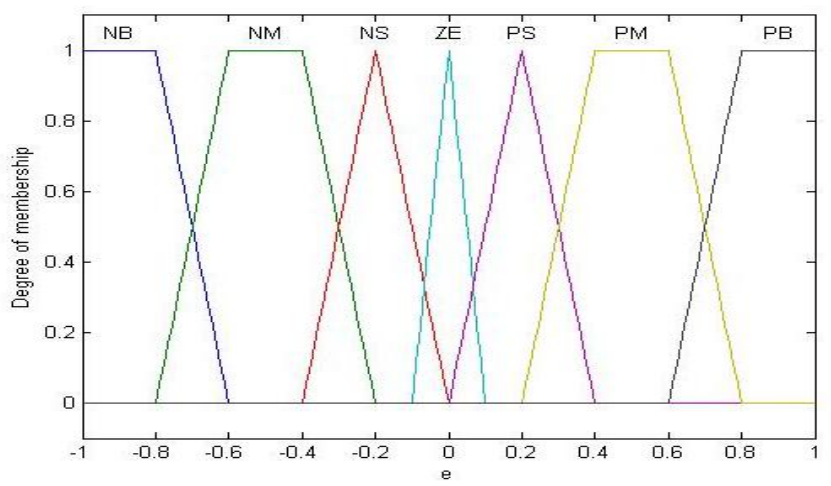

Fig. 7. Modified membership functions.

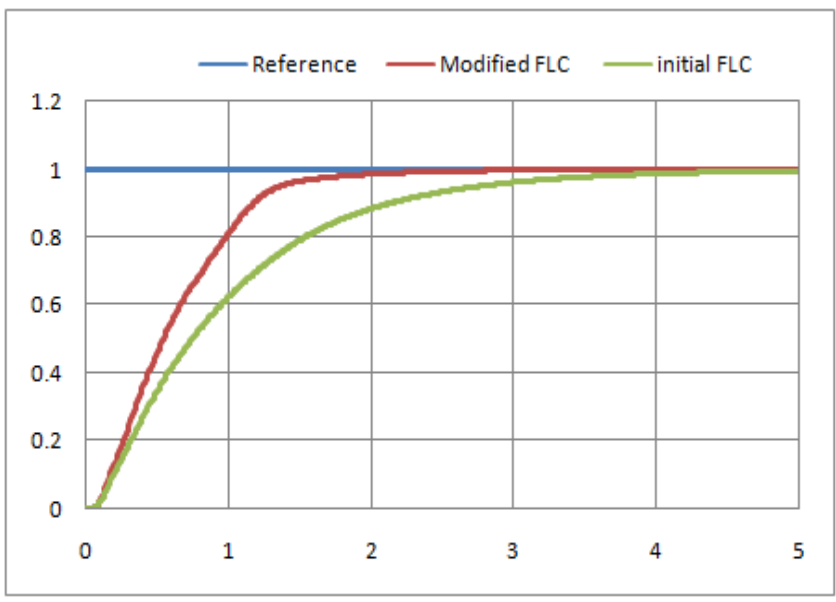

Fig. 8. Step response in Azimuth plan. 


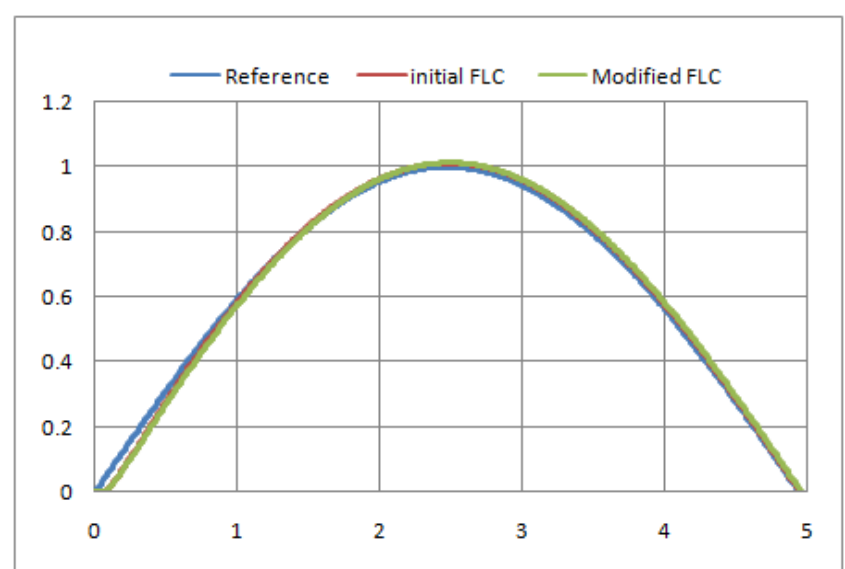

Fig. 9. Sinus response in Azimuth.

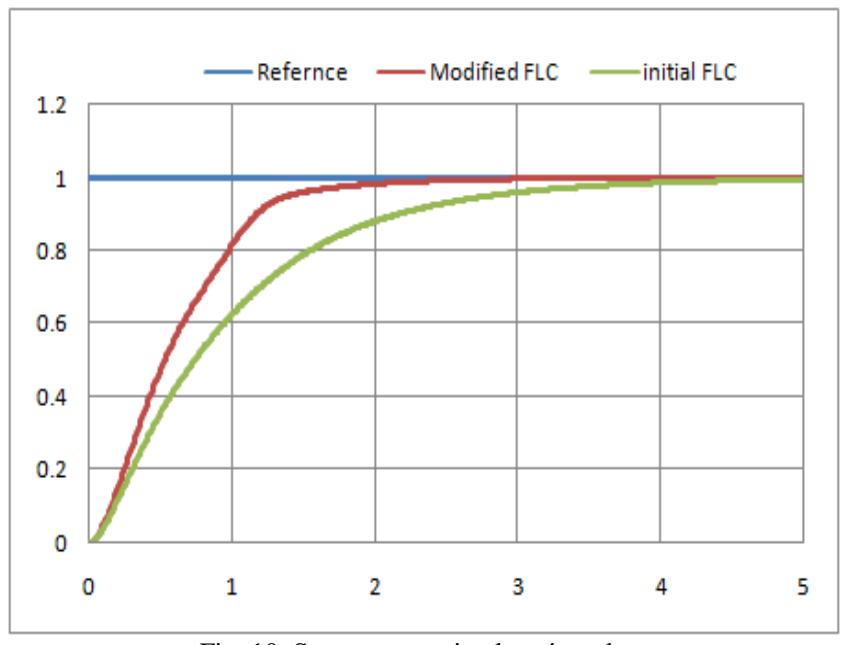

Fig. 10. Step response in elevation plan.

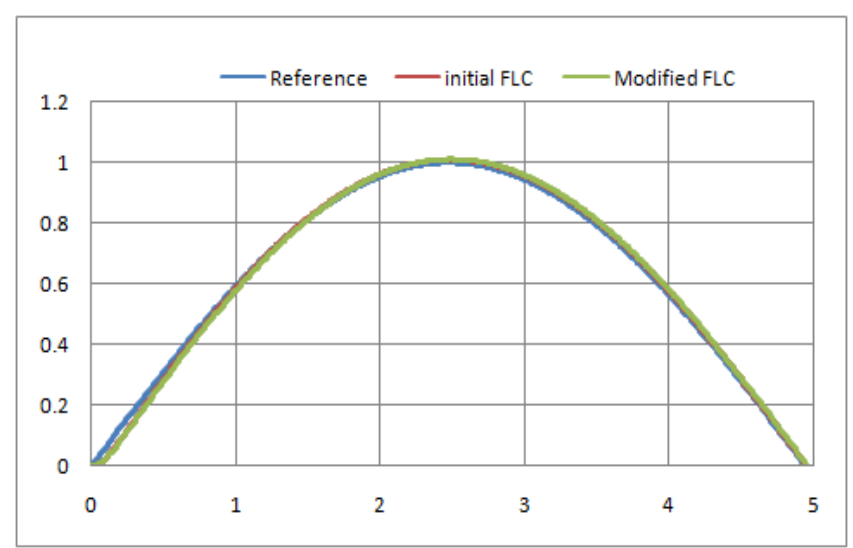

Fig. 11. Sinus response in elevation.

Medium (PM) THEN the change in control signal (CU) is equal to Negative Medium (NM). The matters in defining rules are how many rules should be used and how to determine the relation in IF-THEN statements. Actually, the solutions are based on the experience of a designer or the previous knowledge of the system. The critical point is if there is not sufficient knowledge applied in the design, the result could be drastically bad. The rules are constructed as shown in Table III.

To send out the armature voltage output, the output in the form of fuzzy sets must be converted to a crisp value. This process is called defuzzification. In current article, the center of gravity method is chosen. Fig. 6 illustrates the initial membership functions used by the initial FLC. Seven triangular shape membership functions are used for each of the inputs and output. The efficiency can be improved by adjusting membership functions and rules in procedure.

\section{Procedure 3: Adjusting Membership functions and Rules}

In order to improve FLC performance, rules and member-ship functions are adjusted by making the area of member-ship functions near ZE region narrower to produce finer control resolution. On the other hands, making the area far from $\mathrm{ZE}$ region wider gives faster control response.

Also, the performance can be improved by changing the severity of the rules [21]. After adjusting the rules and membership functions, the final rules and membership functions are obtained as shown in, Table 4 and Figure (7) respectively.

\section{Simulation RESUlts}

Matlab/Simulink software is used to: simulate the turntable's equations of motion, and to make a good comparison between performances of FLC before (initial FLC) and after (modified FLC) adjusting the fuzzy membership functions and rules. Step and half wave sinus signals were applied to the system as a control inputs. Both the controllers' step and sinus responses of the system were given in Figures (8-11) respectively. For this work, rising times and overshot according to the modified FLC are better than in the original one which its performance is out of the control aim. These results illustrate good performance of the system under control of the modified FLC than that under control of the initial FLC.

\section{CONCLUSIONS}

A detailed RCS Lab construction was illustrated and discussed. Then the importance of automatically tracking targets that improves the target's RCS measurements was mentioned. Using Dinavit-Hartenberg and Lagrangian equation to model and drive the equation of motion in both azimuth and elevation plans. After driving the equations of motion of the turntable (RCS's antenna holder), we found that it is a multi-input/output nonlinear system. In this work, the application of fuzzy logic in outdoor RCS Lab based on the expert knowledge is discussed, analyzed and simulated. Two different fuzzy systems have been tested and compared with a crisp decision system. The first one has been developed using an expert knowledge, and as a refinement, the second one was modified according to the recorded videos. Results obtained show better capabilities for the system to improve tracking, then enhancing the real target RCS measurements. This conclusion encourages for implementing these controllers into the real-time RCS' Lab system.

\section{REFERENCES}

[1] S. Challa and G. W. Pulford, "Joint target tracking and classification using radar and ESM sensors," IEEE Trans. Aerosp. Electron. Syst, vol. 37, 2001, pp. 1039-1055.

[2] Y. Boers and H. Driessen, "Integrated tracking and classification: an application of hybrid state estimation," in Proc. SPIE Signal Data Process, Small Targets, vol. 4473, 2001, pp. 198-209. 
[3] N. Gordon, S. Maskell, and T. Kirubarajan, "Efficient particle filters for joint tracking and classification," in Proc. SPIE Signal Data Process, Small Targets, vol. 4728, 2002, pp. 439-449.

[4] M. I. Miller, A. Srivastava, and U. Grenander, "Conditional-mean estimation via jump-diffusion processes in multiple target tracking/recognition," IEEE Trans. Signal Process, vol. 43, 1995, pp. 2678-2690.

[5] D. Lanterman, "Tracking and recognition of airborne targets via commercial television and FM radio signals," in Proc. SPIE Acquis, Track, 1999, pp. 189-198.

[6] S. Herman and P. Moulin, "A particle filtering approach to FM-band passive radar tracking and automatic target recognition," in Proc. the IEEE Aerospace Conference, vol. 4, pp. 1789-1808, 2002.

[7] S. M. Herman, "A particle filtering approach to joint passive radar tracking and target classification," Ph.D. dissertation, US: Graduate College of the University of Illinois, 2002.

[8] H. Jiang, L. Xu, and K. Zhan, "Joint tracking and classification based on aerodynamic model and radar cross section," Pattern Recognition, vol. 47, no. 9, 2014, pp. 3096-3105.

[9] M. J. Er and Y. L. Sun, "Hybrid fuzzy proportional - Integral plus conventional derivative control of linear and nonlinear systems," IEEE Transactions on Industrial Electronics, vol. 48, no. 6, 2001.

[10] H. F. Shao, B. G. Hu, and Z. L. Zhu, "A case study of one-to-two mapping fuzzy PD controllers on inverted pendulum," Fuzzy Systems Conference Proceedings, 1999.

[11] J. W. Park, R. G. Harley, and G. K. Venayagamoorthy, "New external neuro-controller for series capacitive reactance compensator in a power network," IEEE Transactions on Power Systems, vol. 19, no. 3, 2004.

[12] D. Amoozegar, "Dstatcom modeling for voltage stability with fuzzy logic pi current controller," International Journal of Electrical Power \& Energy Systems, vol. 76, 2016, pp. 129-135.

[13] B. M. Al-Hadithi, A. J. Barragán, J. M. Andújar, and A. Jiménez, Chattering-Free Fuzzy Variable Structure Control For Multivariable Nonlinear Systems Applied Soft Computing, vol. 39, 2016, pp. 165-187.

[14] Z. Xu, H. Y. Nan, and C. P. Yuan, "A study of terrain following controller based on back stepping and variable structure control," Intelligent Control and Automation, vol. 5, 2004.

[15] E. Natsheh and K. A. Buragga, "Comparison between conventional and fuzzy logic PID controllers for controlling DC motors," International Journal of Computer Science Issues, vol. 7, no. 5, 2010.

[16] J. K. Zadrozny, "Computing with words is an implementable paradigm: Fuzzy queries, linguistic data summaries, and natural-language generation," IEEE Transactions on Fuzzy Systems, vol.18, no. 3 , 2010.

[17] M. H. F. Zarandi and A. K. Sedehizadeh, "A hybrid approach to develop an interval type-2 fuzzy logic system," in Proc. 2012 Annual Meeting of the North American Fuzzy Information Processing Society.

[18] H. Hagras, "Developing a type-2 FLC through embedded type-1 FLCs," IEEE World Congress on Computational Intelligence.

[19] F. Xue, A. C. Sanderson, and P. P. Bonissone, and R. J. Graves, "Fuzzy logic controlled multi-objective differential evolution," in Proc. the 14th IEEE International Conference on Fuzzy Systems, 2005.

[20] K. Viswanathan and R. Oruganti, "Nonlinear function controller: A simple alternative to fuzzy logic controller for a power electronic converter," IEEE Transactions on Industrial Electronics, vol. 52, no. 5, 2005.

[21] M. Galal, N. G. Mikhail, and G. Elnashar, "Fuzzy logic controller design for gun-turret system," in Proc. the 13th International Conference on Aerospace Sciences and Aviation Technology, 2009.

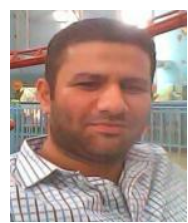

Mohamed G. Mahmoud was born in Lybia on August 20, 1979. He holds a B.E. in communication engineering from MTC in Egypt, and an M.S. in electrical engineering from MTC in Cairo Egypt. He is currently a Ph.D. student in School of Automation at the Nanjing university of science and Technology.

$\mathrm{He}$ is a presently senior engineer at TRC in Egypt. He has held many positions in the past, including graduate research/teaching assistant, communication engineer, and radar cross section measurements/reduction engineer. His current research interests are in the areas RCS measurements, automation and modeling.

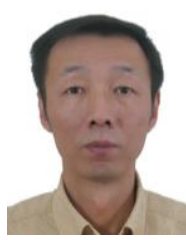

Wang Jianyu was born in China on April 14, 1964. He holds a B.E. in computer science from NJU in China, and an M.S. in system engineering from NJUST in China, and a PhD in computer application from NJUST in China.

$\mathrm{He}$ is a professor and the vice dean of Automation School in NUST. His academic interest lies in systems engineering, computer simulation, emergency management, multimedia information process and some other software-related areas. In recent 10 years he published 34 research papers in the areas of information systems and emergency management. 